La mélancolie de la «vanitas »: des Essais à Hamlet, de Montaigne au Prince Hamlet1 novembre 2003

\title{
Géralde Nakam
}

\section{(2) OpenEdition}

Journals

Édition électronique

URL : http://journals.openedition.org/shakespeare/675

DOI : $10.4000 /$ shakespeare.675

ISSN : 2271-6424

Éditeur

Société Française Shakespeare

Édition imprimée

Date de publication : 1 novembre 2004

Pagination : 221-243

ISBN : 2-9521475-0-7

\section{Référence électronique}

Géralde Nakam, «La mélancolie de la « vanitas » : des Essais à Hamlet, de Montaigne au Prince Hamlet 1 novembre 2003 », Actes des congrès de la Société française Shakespeare [En ligne], 21 | 2004, mis en ligne le 01 novembre 2007, consulté le 19 avril 2019. URL : http://journals.openedition.org/ shakespeare/675; DOI : 10.4000/shakespeare.675 


\section{Shakespeare et Montaigne vers un nouvel humanisme}

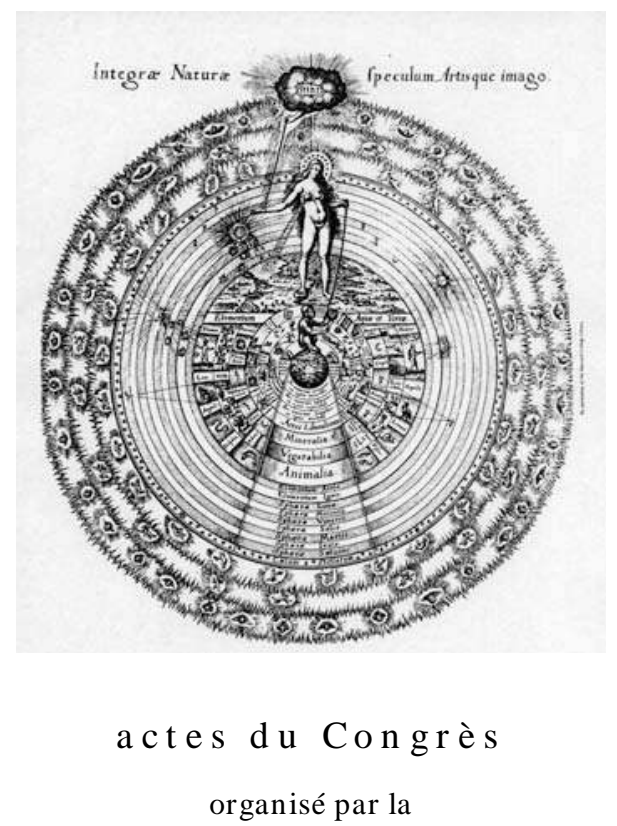

SOCIÉTÉ FR ANÇAISE SH AKESPEARE en collaboration avec la

S OC I É T É I N T E R N A T I O N A L E DES AM I D E M O N A I G N les 13,14 et 15 mars 2003

$$
\begin{gathered}
\text { textes réunis par } \\
\text { Pierre KAPITANIAK } \\
\text { sous la direction de } \\
\text { Jean-Marie MAGUIN }
\end{gathered}
$$




\section{COMITÉ SCIENTIFIQUE :}

Margaret Jones-Davis

Gisèle Venet

Jean-Marie Maguin

Yves Peyré

François Laroque

Pierre Kapitaniak

\section{COUVERTURE :}

Robert Fludd

Utriusque Cosmi Historia (1617-19)

planche 17

conception graphique et logo

Pierre Kapitaniak

\section{(C) 2003 Société Française Shakespeare}

Institut du Monde Anglophone

Université de Paris III - Sorbonne Nouvelle

http:// univ-montp3.fr/SFS/

5 rue de l'École de Médecine

75006 Paris

Diffusion :

AVL DIFFUSION

Parc Euromédecine

34198 MONTPELLIER CEDEX 5

ISBN 2-9521475-0-7

Tous droits de traduction, de reproduction et d'adaptation réservés pour tous les pays 


\section{LA MÉLANCOLIE DE LA « VANITAS »: \\ DES ESSAIS À HAMLET, de Montaigne au Prince H amlet}

Géralde NAKAM

Comme Montaigne, Shakespeare, ou du moins son héros Hamlet, reconnaît en lui-même avec inquiétude le tourment propre aux « mélancoliques " de la Renaissance. Tous deux pratiquent le seul antidote à ce mal, et à sa dérive redoutée, la folie: le gnôthi seauton, qui oriente chaque "essai » de Montaigne, et dont l'intense exigence de lucidité commande aussi les monologues d'Hamlet. Le sentiment de l'absolue "vanitas " de toute chose et d'eux-mêmes, le sens aigu de la mort et sa tentation, ou sa fascination, les habitent tous deux, avec pourtant un amour épicurien de la vie. Chez Montaigne, la vie l'emporte, avec sa liberté, qui s'exerce dans chaque essai, et grâce à son livre, son refuge et son salut. Torturé par l'assassinat de son père, par l'ignominie de sa mère, la mort d'Ophélie, habité par le sens du péché, Hamlet est pris au piège d'un mauvais destin, dans un univers fermé par la prédestination.

The Melancholy of "vanitas": in Montaigne and Hamlet "Melancholy" is to be found both in the works of the essayist and in Prince Hamlet. It emerges in broad shared themes (in particular, those expressed by Montaigne in "De la Solitude", "De la Tristesse", "L'Apologie de R. Sebond", "De la Vanité" etc.) converging on, and inspired by, an awareness of "vanitas" that is equally lucid and fundamental: the emptiness and vanity of man and of human existence, as announced in Ecclesiastes. However, in the two cases which interest us, the suffering of melancholy is identical neither in quality nor in its consequences; nor does the sentiment of "vanitas" play the same role in the poetic and philosophical conceptions governing the two works. The analysis of the present author, that of an ordinary reader, merely seeks to track some of the paths from Montaigne to Hamlet and back again, from the point of view of a "melancholy" which imbues and, doubtless, determines them.

La ressem blance ne fait pas tant un comme la différence fait autre.
Nature s'est obligée à ne rien faire autre qui ne fût dissem blable.
Montaigne, «De l'expérience», III, 13, $1065 \mathrm{~b}, \mathrm{c}^{299}$.

I

$\mathrm{V}$

oici deux personnages en vis-à-vis. Ils pourraient dialoguer ; le second, en tout cas, interroger le premier. Celui-ci, vêtu de noir ${ }^{300}$, se tient seul, à l'écart, dans une assemblée bruyante, et brillante. Ce jeune homme méditatif (il évoque toujours pour moi un Titien) pense à la mort, «qui nous tient au collet» (I, 20, 85a).

${ }^{299}$ Essais, Paris, P.U.F., 1965. Respectivement: le livre, l'essai, la page, la couche d'écriture (a, b ou c). Je modernise la graphie. Pour l'idée, cf. Edith Wharton, qui rapporte, dans Les chemins parcourus (A Backward Glance), ce propos de son ami Walter Berry: «C'est facile de voir des ressemblances superficielles entre les œuvres. Mais il faut un esprit de premier ordre pour distinguer les différences profondes. » (!) coll. 10/18, 2001, p. 116.

300 « Je ne m'habille guère que de noir, ou de blanc, à l'imitation de mon père », Essais, «De l'usage de se vêtir», I, 36, 227a. 
Il observe ces invités joyeux, des morts en sursis, comme lui-même... Des années plus tard, devenu écrivain pour « s'essayer » à comprendre et à maîtriser sa pensée dans le soliloque continu d'un livre, il s'en souvient, et en marque ses premiers autoportraits :

Je suis de moi-même non mélancolique, mais songe-creux. Il n'est rien de quoi je me sois dès toujours plus entretenu que des imaginations de la mort: voire en la saison la plus licencieuse de mon âge, parmi les dames et les jeux, tel me pensait empêché à digérer à part moi quelque jalousie, ou l'incertitude de quelque espérance, cependant que je m'entretenais de je ne sais qui, surpris les jours précédents d'une fièvre chaude et de sa fin au partir d'une fête pareille, et la tête pleine d'oisiveté, d'amour et de bon temps, comme moi, et qu'autant m'en pendait à l'oreille. (I, 20,87a)

Le second, vêtu de noir aussi, dans son «manteau couleur d'encre » - il est en deuil - et au dedans l'âme plus en deuil encore dans sa «couleur de nuit ${ }^{301}$, tient à la main ici un livre, là un crâne, ou une épée. Lui aussi aurait pu être le modèle d'un des portraitistes de la Renaissance, de la manière d'un Bernardino Licinio ${ }^{302}$, ou mieux, d'un Salviati, d'un Pontormo. Enfermé en lui-même, il examine la pensée qui le hante, de soliloque en soliloque: tant sa solitude est totale, car il lui est interdit de se confier. Un mort l'obsède, et sa propre mort. La tentation du suicide l'a saisi et se fait jour dès sa toute première méditation ( O Oh! si cette chair trop consistante pouvait fondre...», I.ii.129) ${ }^{303}$, avant même les révélations du spectre, et jusqu'à la poignante interrogation que l'on sait :

Mourir, dormir -

Pas plus $[\ldots]$

Dormir, rêver peut-être.

(III.i.62-67)

Propos qui semble faire écho à cet autre, du premier :

Si c'est un anéantissement de notre être, c'est encore amendement d'entrer en une longue et paisible nuit. Nous ne sentons rien de plus

\footnotetext{
${ }^{301}$ Ham let, trad. Michel Grivelet, Paris, Robert Laffont, coll. « Bouquins », 1995, I.ii.77 et 68. Toutes les références seront à cette édition.

68. Toutes les références seront à cette édition.
302 Licinio n'aurait pas pu le peindre, vu ses dates (1491-1549), mais son portrait d'un jeune homme vêtu de noir avec un rabat blanc, la main posée sur un crâne, conviendrait si bien au personnage d'Hamlet, qu'il est représenté en couverture de l'éd. d'Oxford de la tragédie.

303 Je préférerais « consistante» ou « ferme », à « solide » proposé.
} 
doux en la vie qu'un repos et sommeil tranquille et profond, sans songe. $(\text { III, } 12,1053 \mathrm{c})^{304}$

Sans le support de ces deux portraits, que j'avais dans l'esprit, je ne me serais pas risquée dans cette gageure, d'une «outrecuidance démesurée ${ }^{305}$, dirait Montaigne, d'oser parler de Shakespeare, du Prince Hamlet, qui plus est, et pire encore, dans un rapprochement a priori aussi incongru peut-être que la rencontre surréaliste «d'une machine à coudre et d'un parapluie sur une table d'opération »...

Qu'y a-t-il de commun, en effet, pour ne prendre que deux éléments (et sans parler de la différence des genres), entre l'étudiant de Wittenberg nourri de luthéranisme, pénétré d'un sens du péché paulinien, augustinien, irréductible, et une pensée que, semble-t-il, le péché ne concerne pas et qui se refuse à évoquer les mystères de l'audelà? Entre la représentation du désastre absolu conçu par Shakespeare à travers la destruction et l'autodestruction de son héros, et, au contraire, les représentations diverses que Montaigne découvre de lui-même, d'essai en essai, pour se connaître et entraîner son lecteur à se construire avec lui, à «se bâtir » - pour reprendre sa métaphore biblique ?306

Rien de commun, donc, sinon deux données: la présence des Essais dans Hamlet ${ }^{307}$, et la «mélancolie». Non seulement Shakespeare a lu Montaigne ${ }^{308}$, mais Hamlet le lit en scène. Ce sont les Essais, je pense, qu'il tient à la main en se promenant dans la galerie où Polonius vient le rencontrer :

304 Rapprochement également relevé (avec prudence) par Robert Ellrodt, «Selfconsciousness in Montaigne and Shakespeare», Shakespeare Survey, vol. XXVIII, 1975, p. 41.

305 III, 11, 1035 b.

306 «Il s'est bâti à diverses poses et intervalles...», dit Montaigne de son livre, dans « De la ressemblance des enfants aux pères » (II, 37, 758a). Sur cette métaphore, voir G. Nakam, Le dernier Montaigne, Champion, 2002, chap. III «Ibériques de Montaigne», p. 83 et la note 43 .

${ }^{307}$ Fait désormais incontestable, je pense, malgré la formule péremptoire de P. Villey selon laquelle plusieurs zéros additionnés font toujours zéro! Voir, déjà, les beaux livres de Clara de Chambrun, Giovanni Florio, un apôtre de la Renaissance en Angleterre à l'époque de Shakespeare (1921), Shakespeare acteur et poète (1926), Shakespeare retrouvé (1947), ou Ham let de Shakespeare (1929).

308 Il a même connu le texte des Essais avant la traduction de Florio, et en français comme les critiques l'ont établi. 
Et il lui lit, sauf erreur, un extrait de « Du Repentir »... façon Hamlet. J'y reviendrai.

Quant à «l'humeur noire» de la Renaissance, dont la Mélancolie trop studieuse, pensive et triste, gravée par Dürer est l'allégorie et l'emblème, elle est, me semble-t-il, depuis Pétrarque d'ailleurs, constitutive d'une époque consciente qu'elle «renaît» sur des ruines et se nourrit de morts. Cette ambiguïté la traverse tout entière, d'un siècle à l'autre de sa diffusion à travers l'Europe.

Définie, mais sans cesse réinterrogée, à travers traités médicaux et problèmes philosophiques, la mélancolie ne s'associe pas seulement au génie créateur, dont elle alimente l'inquiétude ou l'exigence, à son examen constant et répétitif, à sa pensée sur le qui-vive, à sa quête. Elle apporte une alerte en soi, le pressentiment d'une menace, une terreur peut-être, et la conviction, selon les cas, redoutable, paralysante, de la vanité de toute chose.

C'est l'excès de cette humeur fondamentale de toutes les typologies du temps, qui fragilise et peut déséquilibrer la psyché, alors envahie par une souffrance qui va, selon les degrés du mal et son histoire dans l'individu, d'une anxiété tenace à la plus destructrice des détresses.

Pour Aristote, dans le fameux Problème $X X X$, elle est la marque même du génie, et c'est «par nature» et non «par maladie» que le mélancolique est un être d'exception ${ }^{309}$. Un Timothy Bright, au contraire, observant le tourment qui ravage une conscience pénétrée du sentiment de sa faute, s'ingénie à dissocier ce scrupule, moral et religieux, de la mélancolie. L'une, dit-il, est une maladie de l'imagination qui travaille l'être jusqu'à lui faire «perdre le goût de la vie », l'autre concerne la conscience affligée de son péché. L'une relève de la médecine, l'autre de la foi. Cependant, le composé des deux se rencontre, d'autant plus que le mélancolique est plus que quiconque exposé au tourment religieux. Satan même profite de son mal et «s'active» pour jeter dans le désespoir le malheureux, et le porter

309 «tous les mélancoliques sont donc des êtres d'exception, et cela non par maladie, mais par nature». C'est la dernière phrase du traité. Aristote, L'homme de génie et la mélancolie, trad. prés. et notes de Jackie Pigeaud, Paris, Rivages Poche, 1988, p. 107. 
alors à des violences envers soi-même et envers les autres ${ }^{310}$. Shakespeare a lu ce Traité de la Mélancolie, publié en 1586.

Mais d'un autre côté, il est un antidote, précisé par les néoplatoniciens, et notamment par Marsile Ficin, contre l'excès de ce mal et ses périls de perte d'identité, de déchéance morale et mentale, d'évolution fatale vers la folie: le principe socratique du gnôthi seauton $^{311}$, recommandé par l'oracle de Delphes, et par lequel Montaigne termine son essai «De la vanité». Ce principe de sauvegarde est constamment à l'œu ure dans les Essais, il y commande l'auto-analyse et l'invention de l'autoportrait. Il inspire et conduit aussi les monologues qui scandent si admirablement la tragédie d'Hamlet, la méditation intérieure et tourmentée de son héros.

Introspectifs, imaginatifs (penser à «De la force de l'imagination» de Montaigne ${ }^{312}$ ), grands liseurs de livres anciens et modernes, nos personnages sont instruits des théories en vogue sur la mélancolie : nature ou maladie ? si maladie, selon quels degrés, dans quelles proportions du mélange des humeurs? génie ou culpabilité ? Dans le miroir de ces conceptions, ils se voient, se craignent mélancoliques. Leur époque tumultueuse et déchirée les y invite aussi. Montaigne et Shakespeare n'ont pas seulement en commun les sources livresques de leur érudition, et le même livre des Essais, mais cette «melancholia» en eux dans laquelle ils lisent et se sourcent, pour la comprendre, peut-être la combattre, ou y succomber. L'alchimie de leur mélancolie, dans l'alambic de leur texte, n'est pas moins subtile, complexe, dépendante des mentalités contemporaines, mais pour chacun singulière, que celle de leurs lectures et de leur savoir ${ }^{313}$.

310 Timothy Bright, Traité de la Mélancolie (1586), trad. et prés. par Éliane Cuvelier, Grenoble, Éditions Jérôme Millon, 1996, respectivement chap. 8, p. 67; chap. 35, p. 204 ; chap. 36, p. 223, 224 et 228.

311 Voir Robert Klein, La forme et lintelligible, Paris, Gallimard, 1970.

312 Titre, aussi, d'un chapitre de Robert Burton, Anatomie de la mélancolie, trad. de Bernard Hoepffner et Catherine Goffaux, Paris, José Corti, 2000, vol. I, p. 424. Burton a lu Montaigne, dont il cite plusieurs essais et exemples.

$313 \mathrm{Il}$ y a bien d'autres rapprochements à examiner entre les Essais et Hamlet, que je me borne à proposer ici : mensonge de la rhétorique, haine de la certitude, cruauté, vengeance, pouvoir du théâtre, etc. 
Hamlet, comme Montaigne, identifie en lui ce mal. Il s'en inquiète. Montaigne, on l'a vu, nuance: non pas vraiment «mélancolique», mais rêvasseur, «songe-creux» (I, 20, 87a). Il situe, avec une pointe d'humour, sa complexion «entre le jovial et le mélancolique» (II, 17, $641 \mathrm{~b})$ : entre Jupiter et Saturne... S'agit-il d'équilibre ou d'in stabilité ? S'il se défend de céder à la complaisance de cette humeur ${ }^{314}$, c'est qu'il craint son pouvoir néfaste, comme il ressort de l'essai «De la tristesse », le second de ses chapitres, où, tout en détestant le mal de la «tristezza », qui est perverse, il décrit si fortement, de façon si intime, «cette morne, muette et sourde stupidité qui nous transit» (I, 2, 12a), qu'il est impossible de ne pas y lire une détresse vécue, ou du moins approchée de près en lui-même $\mathrm{e}^{315}$.

Sa thérapeuthique de la «diversion », qu'il analyse dans l'essai de ce nom, c'est à Rome qu'il en a vérifié le bienfait, compris le mécanisme. Dans son Journal de voyage, il note :

Je n'ai rien si ennemi à ma santé que l'ennui et l'oisiveté : là [à Rome], j'avais toujours quelque occupation, sinon plaisante, que j'eusse pu désirer, au moins suffisante à me désennuyer...

Tous ces amusements m'embesognaient assez: de mélancolie, qui est ma mort, et de chagrin, je n'en avais nulle occasion, ni dedans, ni hors la maison 316 .

La diversion du voyage est plus forte que celle des livres. Mais la plus essentielle, car, en plongeant au cœur du mal, elle l'analyse et permet, peu ou prou, de s'en libérer, c'est la diversion de l'écriture. Montaigne confie, au début de «De l'affection des pères aux enfants » :

C'est une humeur mélancolique, et une humeur par conséquent très ennemie de [dangereuse pour] ma complexion naturelle, produite par le chagrin de la solitude en laquelle il y a quelques années que je m'étais jeté, qui m'a mis premièrement en tête cette rêverie de me mêler d'écrire. Et puis, me trouvant entièrement dépourvu et vide de toute autre matière, je me suis présenté moi-même à moi, pour argument et

\footnotetext{
314 II, 20, B, 674, «Nous ne goûtons rien de pur » : «J'imagine bien qu'il y a du dessein, du consentement et de la complaisance à se nourrir en la mélancolie...»

$315 \mathrm{Je}$ ne reprends pas ici mon étude «La mélancolie et la folie », in Montaigne, la Manière et la matière, Paris, Klincksieck, 1992, chap. 2, p. 31 et suiv., auquel je me permets de renvoyer

316 Voir Montaigne, La Manière et la matière, p. 41.
} 
pour sujet. C'est le seul livre au monde de son espèce, d'un dessein farouche et extravagant. (II, 8, 385a, c)

Tous les Essais sont là : la solitude du sujet, son effort pour se sauver d'un péril mortel, la décision de l'autoportrait - geste volontaire : «Je me suis présenté à moi...»- et l'étrangeté radicale de ce livre.

Hamlet a changé317. Il le constate en lui. Son entourage le souligne: Claudius, sa mère, Ophélie s'en montrent soucieux. Il leur répond avec des sarcasmes de souffrance indicible. Son «âme prophétique » lui soufflait la vérité. Après les révélations du spectre, c'est au faux ami Guildenstern qu'il confie son accablement: "J'ai, insiste-t-il, perdu toute ma gaieté, délaissé mes exercices » (II.ii.275-6). Qu'il commence ou non déjà à jouer la comédie, son changement reste évident. Mais, pour éprouver de façon si exacte sa mélancolie, il y était prédisposé, ou plutôt, elle lui est familière. À la fin du même acte II, il en est si tourmenté qu'il redoute que le spectre ne soit un démon profitant de son mal pour s'emparer de lui et le damner, comme le rappelait $\mathrm{T}$. Bright, «mettant à profit ma faiblesse et ma mélancolie » (II.ii.544-5). Plus loin, Claudius, dont la haine est perspicace, loin d'être dupe de sa « folie », en pénètre la nature et comprend le danger qu'elle recèle pour lui : " Il y a dans son âme quelque chose que sa mélancolie est en train de couver» (III.i.159-60). Hamlet enfin, à l'acte V, en menace Laërte :

J'ai pourtant quelque chose en moi de dangereux

Que tu ferais sagement de craindre.

(V.i.228-9)

La solitude, la tristesse, sont creusées par le décalage que, comme Montaigne, Hamlet ressent avec son temps et son pays. Ce «temps malade», dont le diagnostic s'alourdit dans les Essais, d'édition en édition au rythme chaotique des guerres de Religion, Montaigne, en «discordance» radicale, le décrit, en particulier dans « De la Physionomie » et « De la Vanité », en termes de décomposition, de gangrène, d'amputations. Ce sont, dit-il, «ulcères envieillis». Le pays est menacé de «dissipation» et de «divulsion»: poussière, arrachement, démembrement ${ }^{318}$. Sa nostalgie de temps plus nobles et

${ }^{317}$ Hamlet n'est pas du tout un «atrabilaire», je suis bien, sur ce point, de l'avis de V. Bourgy, Shakespeare, Tragédies, vol. I, p. 259, art. « Mélancolie» du «Dictionnaire de Shakespeare».

318 Respectivement III, 12, C, 1047 ; III, 9, C, 962 et III, 12, C, 1043. 
plus sincères, «le temps de nos pères », n'en est que plus ardente. Comme l'Antiquité s'idéalise, le père de Montaigne incarne une perfection.

Des contrepoints similaires se trouvent chez Hamlet, dans un Danemark en état d'alerte de guerre - guets, fabrication intense et achats d'armes, «préparatifs fiévreux »: donnée mise en relief dès la première scène, où le lucide Marcellus détecte une annonce «d'éruption», dont l'apparition du spectre serait le présage, pour affirmer un peu plus loin le célèbre: «Quelque chose est pourri dans l'état du Danemark» (I.iv.67) ${ }^{319}$. Reprise à l'acte v par le fossoyeur, qui associe à la situation du Danemark la décomposition des corps (v.i.13543), l'image de la pourriture borne ainsi de part et d'autre la pièce entière.

En contraste, et surtout en contraste avec Claudius, cause et représentant de ce mal, Hamlet exalte les vertus et la beauté d'un père, plus encore qu'idéalisé, divinisé (il le compare à tous les dieux de l'Olympe), et en même temps pleinement homme :

Voyez quelle grâce régnait sur ce visage-ci, [...]
Une harmonie et véritablement une forme
Sur laquelle chaque dieu semblait mettre son sceau
Pour garantir au monde que c'était là un homme.

(III.iv.54-61)

La pureté et la noblesse d'Hamlet, merveilleusement décrites par l'amour d'Ophélie (elle dresse de lui un portrait lyrique : «Miroir d'élégance, modèle de conduite, / Point de mire des regards...»), et par l'amitié d'Horatio (dans son « requiem»), sont exposées au cynisme d'un Claudius, à la veulerie des courtisans. Claudius le sait, et va s'en servir : Hamlet est si confiant, promet-il à Laërte, qu'il ne se doutera d'aucun piège, il ne pensera même pas à examiner les fleurets du duel.

Une même éthique de loyauté et de confiance, de sens de l'amitié, de générosité habite Montaigne, qui est, assure-t-il, incapable de compromissions :

Celui qui va en la presse, il faut qu'il gauchisse, qu'il serre ses coudes, qu'il recule ou qu'il avance, voire qu'il quitte le droit chemin, selon ce qu'il rencontre...(III, 9, 991b)

319 «Ce temps est mal en point» (« out of joint»), dira plus loin Hamlet (I.v.189). 
Ils sont tous deux incapables de telles danses de l'opportunisme. Étrangers à leur monde, étrangers aux commerces ordinaires du monde, ils le sont aussi par leur solidarité avec les morts, et leur intimité avec la mort. Leur grande piété filiale se traduit en tendresse et pitié bouleversantes. Montaigne, dans « De la Vanité »: «Le soin des morts nous est en recommandation. » Les héros antiques ne sont plus?

Ils sont trépassés. Si est bien mon père, aussi entièrement qu'eux, et s'est éloigné de moi et de la vie autant en dix-huit ans que ceux-là ont fait en seize cents; duquel pourtant je ne laisse pas d'embrasser et pratiquer la mémoire, l'amitié et société, d'une parfaite union et très vive. (III, 9, 996b)

La hantise du meurtre de son père (aggravée par la traîtrise et la débauche sexuelle de sa mère) fait d'Hamlet un fils hanté par ce père vénéré, rempli de pitié pour son spectre torturé dans les souffrances du Purgatoire, et qui a tant besoin de son aide. Le message du roi Hamlet habite son fils (il n'aura plus jamais en tête d'autres livres, jure-t-il, que ces paroles), comme sans doute Shakespeare fut habité par le «testament» de son propre père demandant prières et actes expiatoires, « de crainte qu'en raison de mes péchés, je sois conduit et demeure longtemps au purgatoire ${ }^{320}$. Les fils le savent : leur souvenir sauve leur père d'une mort totale. «Remember!» supplie le roi Hamlet. Son fils le lui promet :

Me souvenir de toi ?

Oui, pauvre spectre, tant que durera la mémoire

En ce globe égaré. Me souvenir de toi?

Oui, j'effacerai des tablettes de ma mémoire

Tout ce qui y fut inscrit de futile et de sot. [...]

Et ton commandement sera le seul à vivre

Dans ce livre, ce volume de mon cerveau..

$(\text { i.v. } 95-103)^{321}$

La mélancolie choisit, ici et là, de s'exprimer à travers des thèmes repérés depuis l'Antiquité et surtout depuis l'Ecclésiaste : le savoir, la

320 Cité par Jean-Marie et Angela Maguin, William Shakespeare, Paris, Fayard, 1996, p. 38. Article XII du « Testament».

${ }^{321}$ Sur la conception du Purgatoire et le culte du souvenir des morts, voir le très beau Hamlet in Purgatory de Stephen Greenblatt, Princeton University Press, 2001, que je remercie vivement Nick Myers et Jean-Marie Maguin de m'avoir indiqué. 
femme, l'action humaine, les âges de la vie, la vieillesse et sa déchéance, le Temps, la mort. Je m'arrêterai sur trois d'entre eux, pour suggérer, cette fois, la transformation qui s'opère d'un texte à l'autre.

Dans le chapitre «De la Présomption», Montaigne montre au lecteur une blessure originelle, jamais cicatrisée : son irrésolution :

Je ne veux donc pas oublier encore cette cicatrice, bien mal propre à produire : c'est l'irrésolution, défaut très incommode à la négociation des affaires du monde [...]. Je sais bien soutenir une opinion, mais non pas la choisir [...]. L'incertitude de mon jugement est si également balancée en la plupart des occurrences que je compromettrais volontiers à la décision du sort et des dés...(II, 17, 653-654a)

Et Hamlet :

Et la couleur innée de la résolution

Pâlit sous la pensée qui la rend maladive ;

Alors les entreprises capitales et de poids,

Détournant leur courant à voir ce qu'il en est,

Perdent le nom d'action...

(III.i.86-90)

Certes, Montaigne insiste sur son «jugement», et Hamlet sur l'action. Mais c'est toujours la vivacité, l'intensité, l'exigence de la réflexion qui freine et paralyse l'action. C'est du moins ce qu'ils éprouvent. Car, en fait, Montaigne est magistrat, Parlementaire, homme politique, maire de sa ville sous deux mandats, écrivain célèbre. Hamlet met en place sa souricière, sermonne sa mère, retourne contre les émissaires de Claudius le piège fomenté contre lui. Pourtant, il faillit à sa mission, il ne venge pas son père au sens strict. Il semble s'enliser dans les reproches qu'il se fait, dans les débats que lui impose son destin, dans la rationalité qu'il y cherche mortellement. En tout cas, il s'impute à crime les délais de ce qu'il tient pour son devoir. Si sa mélancolie le rend irrésolu, cette indécision même l'empire. Montaigne fait de son défaut un principe de pensée critique, antidogmatique, un outil scientifique pour l'analyse : le « DISTINGO » (II, 1, 335b).

La peur de la déchéance de la vieillesse inspire à Montaigne des lignes cruelles sur ses « grimaces difformes et pitoyables », dans «Sur des vers de Virgile » (III, 5, 893b), et surtout à la fin de «Du Repentir » sur «ses yeux chassieux et troubles», ses «catarrhes» et sa «colique ». Il écrit : 
Elle nous attache plus de rides en l'esprit qu'au visage; et ne se voit point d'ames, ou fort rares, qui en vieillissant ne sentent à l'aigre et au moisi. (III, 2, 817b)

(Montaigne, on le sait, est sensible aux odeurs : sa métaphore est olfactive !) Tel est, je crois bien, le miroir qu'Hamlet tend ironiquement au détesté Polonius :

- Que lisez-vous, monseigneur ?

- Des mots, des mots, des mots.

- De quoi s'agit-il, monseigneur?

[...]

- De calomnies, monsieur ; car ce coquin d'auteur satirique dit ici que les vieux ont la barbe grise, la face ridée, que leurs yeux distillent une résine épaisse, ou de la gomme de prunier, qu'ils ont un plantureux manque d'intellect avec d'extrêmement faibles jarrets...(II.ii.188-96) ${ }^{322}$

L'allégorie repoussante proposée par Montaigne inspire ces sarcasmes. L'imagination de la matière chez Shakespeare, une imagination visuelle et tactile, sait voir dans des yeux «chassieux» des yeux dégoulinants d'une humeur visqueuse, qu'elle appelle résine ou gomme de prunier $^{323}$.

Le suicide fascine Montaigne dès l'origine, avec des variations, toutefois. Dès l'« Apologie », il recommande «d'endormir » et «pallier la plaie » (II, 12, 495) : d'anesthésier la souffrance, de la supprimer par la mort, « le non-sentir et le non-être. » (II, 12, 496a). Il lui consacre le chapitre «Coutume de l'île de Céa » et prononce :

La mort est la recette à tous nos maux [...]. La plus volontaire mort, c'est la plus belle...(II, 3, 351a)

Cette pensée revient à plusieurs reprises (I, 20 ; I, 14, etc.). Mais ce que Montaigne célèbre plus que tout, et de plus en plus après 1588 , c'est le

322 Je préférerais «rogue », fripon, coquin, maraud, à «slave», et de toute façon pas "pleutre». À propos de Polonius: il incarne tout ce que Montaigne déteste autant que Shakespeare, à savoir, la certitude («'Tis so »!), la méfiance (il fait espionner son fils; il ne donne à Ophélie que des leçons de méfiance). Mais Shakespeare joue aussi avec ce personnage, qui, assure-t-il, a joué jadis le rôle de Jules César: comme l'avait fait Montaigne au Collège de Guyenne...(Voir I, 26, 176b).

323 Florio traduisait : «bleare and troubled eyes », Montaigne's Essays, Everyman Library, 3 vol., vol. III, p. 36. Ce rapprochement inédit (sauf erreur et ignorance de ma part) me semble en tout cas mériter examen. Toutefois, comme le rappelle François Maguin dans son édition d'Hamlet (Garnier-Flammarion, 1995, p. 450) d'après Harold Jenkins dans son Hamlet (Londres, The Arden Shakespeare, 1982, p. 467), les hypothèses sur le livre d'Hamlet vont des Satires de Juvénal à l'Éloge de la Folie d'Érasme... Voir aussi la note 51 à la fin de cette étude. 
choix héroïque de la mort volontaire pour échapper au tyran, lui voler sa victoire : héros solitaires, comme la noble Theoxena en Thessalie, ou communautés entières, comme les habitants d'Astapa en Espagne ${ }^{324}$. C'est pour la liberté que Montaigne justifie et glorifie le suicide. Car pour la vie elle-même, il ne la hait ni ne la dédaigne -

Car enfin c'est notre être, c'est notre tout [...] c'est contre nature que nous nous méprisons [...] c'est une maladie particulière et qui ne se voit en aucune autre créature, de se haïr et dédaigner. (II, 3, 353a)

- leçon elle aussi reprise et amplifiée en 1588, partout où Montaigne dénonce le mépris et la haine de soi et de la vie, en particulier dans «Sur des vers de Virgile» et « De l’Expérience». Le suicide est, lui, un acte de résistance politique.

Mourir pour ne plus rien sentir des méchancetés du monde, c'est la toute première et constante tentation d'Hamlet depuis, en tout cas, la mort de son père. Son tadium vitce se traduit en images désolées d'un monde en friche grossière envahi de mauvaises herbes. Il se supprimerait dès ce moment, affirme-t-il dès la scène ii de l'acte $\mathrm{I}$, où il apparaît, tenant sa vie pour rien, se souciant de lui «comme d'une guigne», sans l'interdiction divine du suicide. La vie ? «Une mer d'épreuves » : le mépris des gens, le triomphe de l'oppresseur, l'amour dédaigné, la morgue des puissants. C'est pure poltronnerie (« cowards ») que de continuer à vivre. Le frein unique est la terreur de l'au-delà : «the dread of something after death » (III.i.80).

Mais tout le comportement d'Hamlet est suicidaire. Suivre un spectre dans une nuit glacée jusqu'au bord de l'abîme. Jouer la comédie de la folie, péril extrême pour la raison : voir l'avertissement de l'essai «De ne contrefaire le malade» (II, 25) ${ }^{325}$. Mettre en place une souricière dont, de toute façon, il mourra, que soit ou non révélé le crime de Claudius et de sa mère ${ }^{326}$. S'élancer dans la fosse d'Ophélie geste révélateur - en proclamant les droits de son amour :

\footnotetext{
${ }^{324}$ Respectivement II, 27, 699c et II, 3, 361c. Sur la problématique de la tyrannie et du suicide, voir G. Nakam, Le dernier Montaigne, chap. 2, «L'autoportrait d'une liberté ».

${ }^{325}$ Sur le risque de devenir fou, bien analysé par Montaigne, voir le chap. «La mélancolie et la folie », dans Montaigne, la Manière et la matière, chap. 2, cité plus haut.

326 Ou la révélation le tuera, ou le doute, ou, surtout, la vengeance du criminel dévoilé (ce qui arrivera).
} 
J'aimais Ophélie. Quarante mille frères ne sauraient, Avec tout ce qu'ils peuvent avoir d'amour, atteindre À la somme du mien.

Accepter enfin son duel avec ce même frère d'Ophélie, Laërte, malgré son pressentiment, qu'il confie à demi à Horatio :

- [...] Mais tu ne saurais croire combien tout, là près du cœur - mais qu'importe.

- Au contraire, mon bon seigneur.

- Ce n'est que sottise, mais c'est une sorte d'appréhen sion qui

troublerait peut-être une femme.

C'est ainsi à Laërte qu'il délègue le soin de le tuer, de se défaire sans péché mortel de sa pesante vie, de réaliser son profond désir de mort et de néant.

Car là où Montaigne se prépare, de longue main, à la mort -

Et me rechante sans cesse: Tout ce qui peut être fait un autre jour le peut être aujourd'hui [...]. Il faut être toujours botté et prêt à partir... (I, 20,88c, a)

- «botté », comme pour un voyage en somme - Hamlet, soumis à la Providence, mais dans un geste de liberté «défiant les augures », fait plus que de consentir à la mort, il la provoque, dans un pari stö̈cien d'apparence, avec un fatalisme ironique :

Nous défions les augures. Il y a une providence spéciale dans la chute même d'un moineau. Si c'est maintenant, ce n'est pas à venir. Si ce n'est pas à venir, ce sera maintenant. Si ce n'est pas maintenant, cela viendra pourtant. Le tout est d'être prêt. (v.ii.150-2) (27 $^{327}$

Que cette gouaille ne fasse pas illusion! Le rire même peut être mélancolie, comme le dira Robert Burton et comme l'impliquait aussi, chez Montaigne, l'essai « De Democritus et Heraclitus ».

Solitude, repliement sur soi, nostalgie, souffrance, la mélancolie du personnage joue dans la genèse de l'œuvre aussi et dans l'action dramatique un rôle important, et d'autant plus fort que, dans la

327 Sur la Providence et la chute d'un moineau, comparer Montaigne, II, 12, 529c, la feuille d'un arbre: «Comme si ce lui était plus et moins de remuer un empire ou la feuille d'un arbre, et si sa providence [celle de Dieu] s'exerçait autrement, inclinant l'événement d'une bataille, que le saut d'une puce...» 
tragédie, évidemment, mais aussi dans les Essais, tous les éléments se nouent étroitement.

Elle exige, je l'ai dit, l'introspection du gnôthi seauton; chez Montaigne, elle appelle l'autoportrait. Ici et là elle se manifeste (car elle n'a rien de flegmatique ni de mou) dans la force même et la rigueur de la pensée, et dans la volubilité des paroles. Elle a une fonction de moteur. Chez Montaigne, elle est génératrice de l'œuvre dans son étrangeté même, et, au-delà, elle permet à Montaigne de se «faire », et d'être pleinement. Dans Hamlet, elle accompagne les progrès de l'action, avec un rôle paradoxal de ressort et de frein pour le prince Hamlet, mais décisif en fait. D’une part, il invente sa propre comédie de la folie - masques de théâtre, révélateurs du vrai, et portant bien la marque de son «humeur noire». D'autre part, c'est bien son trop de lucidité qui l'englue, mais sa mélancolie appelle, par la menace qu'elle « couve », le dernier stratagème de Claudius, par lequel, enfin, l'action s'accélère jusqu'à son dénouement.

Sur le plan esthétique enfin, comme elle a l'exigence du génie, elle noue la prospection et invente la «manière » propre à chaque essai de Montaigne. Dans Ham let, elle commande les monologues du Prince, qui pourraient être, comme on l'a remarqué, autant d'essais à la Montaigne sur le suicide, la mort, la femme, etc. ${ }^{328}$ : ces explorations par le héros de son drame intérieur, qui, en interrompant de place en place le cours cruel des choses, en faisant contraste avec son implacable cruauté, par leur élévation spirituelle ${ }^{329}$, donnent à cette tragédie sa tonalité unique ${ }^{330}$.

Plus que tout autre mélancolique, peut-être, Hamlet est convaincu de la vanité des choses : de l'action, de l'amour, de l'amitié, des livres, des titres, de la mort. Il n'a plus foi en la vie. Le monde et sa splendeur? «Promontoire stérile», «pestilentiel agrégat de vapeurs». L’homme, «ce chef-d'œuvre», «le parangon des animaux»? Une « quintessence de poussière »(II.ii.277-84). Ces décalages sont la première expression

328 Point intéressant souligné par Harold Fisch, « Hamlet's meditation and Montaigne», in Le Réel dans la littérature et la langue, Actes et Colloques, Klincksieck, 1967, p. 287 (résumé).

${ }^{329}$ Harold Fisch, art. cit., où il renvoie à L. L. Martz, The Poetry of Meditation, 1954.

330 Souligné par Clara de Chambrun et par Stephen Greenblatt, entre autres. 
du désenchantement d'Hamlet. À noter que le dithyrambe (ironique) sur l'homme ne se trouve nulle part chez Montaigne, mais reprend, en le raillant (car Hamlet y a cru et fait part là d'une grave déception personnelle), soit l'optimisme d'un Sebond, soit plus directement, comme on l'a noté, celui d'un Pierre de La Primaudaye ${ }^{331}$, en tout cas un humanisme idéaliste et confiant. Montaigne, au contraire, sans illusion sur l'homme à l'heure des Essais, propose, sur ce chapitre, deux échelles pour rabattre sa présomption. Il le ramène au niveau des autres créatures. Il lui montre, par rapport à Dieu, son néant. «C'est un homme: qu'est-il plus caduc, plus misérable et plus de néant?» écrit-il dans «De l'Ivrognerie» (II, 2, 346a). Dans l'« Apologie de Raimond Sebond», il martèle: l'homme est «la pire espèce du monde », il n'a en partage que « inanité, vanité, fénéantise »:

De toutes les vanités, la plus vaine, c'est l'homme.

Et encore :

La plus calamiteuse et la plus frêle de toutes les créatures, c'est l'homme, et quand et quand la plus orgueilleuse. Elle se sent et se voit logée ici parmi la bourbe et le fient du monde, attachée et clouée à la plus morte et croupie partie de l'univers [etc.]...

Plus loin encore :

Qui verra l'homme sans le flatter, il n'y verra ni efficace [valeur], ni faculté qui sente autre chose que la mort et la terre ${ }^{332}$.

La mort est gagnante. Chez Montaigne, l'homme se définit par sa condition de mortel. La vie n'est qu'une succession de morts, rappelle-t-il, à l'issue de la même «Apologie», et par la bouche de Plutarque, dans un étonnant double mouvement contraire, régressif pour l'être vivant, progressif pour le temps :

La fleur d’âge se meurt et passe quand la vieillesse survient, et la jeunesse se termine en fleur d'âge d'homme fait, l'enfance en la jeunesse, et le premier âge meurt en l'enfance, et le jour d'hier meurt en

${ }^{331}$ Pierre de La Primaudaye, dans L'Académie Française : passage cité et traduit en anglais dans Hamlet, A Norton Critical Edition, éd. Cyrus Hoy, W.W. Norton, 2e éd., 1991, p. 118 "I cannot marvel enough at the excellency of Man, for whom all these things were created, [etc.]. »

332 Respectivement II, 12, 448a ; 449a ; 452a ; 554c. C'est moi qui souligne. 
celui d'aujourd'hui, l'aujourd'hui mourra en celui de demain ; et n'y a rien qui demeure ni qui soit toujours un. (II, 12, 602a) $)^{333}$

Mais c'est encore une fois pour fustiger l'homme de sa folle et criminelle prétention de vouloir définir et enfermer dans des dogmatismes Dieu, seul maître du Temps, éternel.

Hamlet est, lui, obsédé par la décomposition du cadavre, par le ver dévoreur de charognes, dont il rappelle à plusieurs reprises le travail efficace ${ }^{334}$. Le cycle de la mort physique exposé à l'acte IV, par la parabole du ver et de l'Empereur, reparaît, sous une forme encore plus sarcastique, dans le syllogisme du bouchon de tonneau, à l'acte v, dans la scène du cimetière.

Ces deux images-symboles, pourtant, viennent de Montaigne, la première à coup sûr. Toujours dans l'« Apologie », Montaigne rappelle un lieu commun sur la vanité des grands et le cours de l'Histoire, qui dépendent parfois...d'insectes :

les poux sont suffisants pour faire vaquer la dictature de Sylla ; c'est le déjeuner d'un petit ver que le cœur et la vie d'un grand Empereur. $(\text { II, 12, 462a })^{335}$

Dans Hamlet, cela devient (Hamlet vient de daguer par erreur Polonius, qui gît derrière un rideau) :

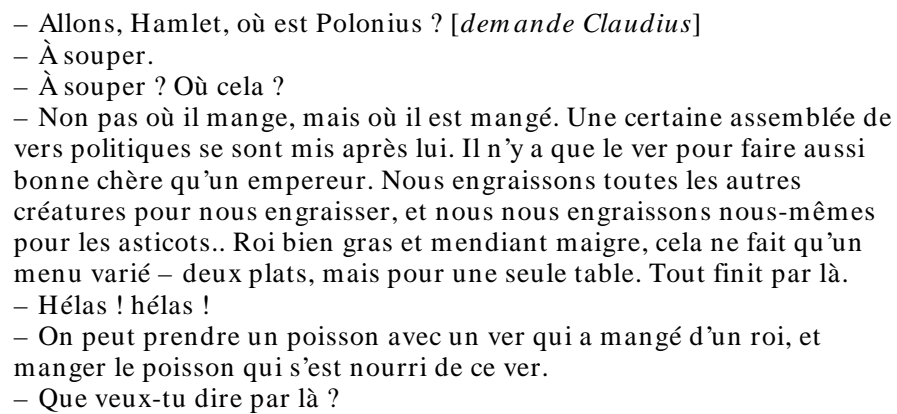

333 Texte de Plutarque, dans la traduction d'Amyot, dont Montaigne n'a pas besoin de préciser l'auteur : tous ses lecteurs d'alors l'identifiaient immédiatement.

${ }^{334}$ Hamlet: "Car si le soleil engendre des asticots dans un chien mort, la charogne étant bonne à baiser - avez-vous une fille ? - Ne la laissez pas au soleil...», (II.ii.180-3). Et dans la scène du cimetière : « et maintenant tout à Gente Dame du Ver...» (v.i.73).

335 Rapprochement relevé par R. Ellrodt, art. cit. 
- Rien, sinon vous montrer comment un roi peut se rendre en voyage officiel dans les tripes d'un mendiant. (IV.iii.17-30)

De l'incise satirique du topos de Montaigne, Hamlet, dans une fabuleuse mise en scène, et poussant jusqu'au bout la logique de son paradoxe, fait une sarcastique, agressive, macabre, drôlissime et grandiose vision de l'œuvre égalitaire de la mort.

Et qui sait si ce n'est pas «la bourbe» de Montaigne ( la bourbe et le fient du monde », cité plus haut), qui se retrouve en argile $\mathrm{du}$ bouchon de tonneau, que devient, cette fois, Alexandre en personne?

À quels bas usages, Horatio, nous pouvons retourner! Pourquoi ne suivrait-on pas en imagination la noble poussière d'Alexandre jusqu'à la voir boucher un trou de bonde?

[...

Alexandre mourut, Alexandre fut enterré, Alexandre retourne en poussière, la poussière est de la terre, de la terre se fait le torchis et pourquoi, avec l'argile qu'il est devenu, ne pas boucher un baril de bière?

Mort, l’impérial César, en argile changé,

Pourrait, bouchant un trou, empêcher l'air d'entrer. (v.i.168-78)

La dérision d'Hamlet, dans une logique rigoureuse comme un enchaînement de syllogismes, et, comme on l'a dit plus haut, par la force visuelle de l'imagination $\mathrm{du}$ poète, transforme dans son développement un mot, une image, une formule, pour, ici, donner à sa raillerie en forme de comptine toute l'intensité de sa douleur, et tout son poids philosophique.

Laquelle philosophie se confond avec toute une poétique de la «vanitas », dont les emblèmes, implacablement matérialisés, sont des crânes, la terre, la poussière ou l'argile, la vermine, le néant, et la figure $\mathrm{du}$ fou. Montaigne transfère son propre et essentiel sentiment de la «vanitas» dans cette paraphrase de L'Ecclésiaste qu'est l'essai « De la Vanité ». Celui-ci est, de tous les Essais, le chapitre qui mêle en lui le plus de tonalités diverses, comme le texte de Salomon, sous l'égide duquel il se place, dès la première ligne: humour et désespoir, amertume et sérénité, satire, dérision. Tout est vanité, soit : mais les beaux préceptes aussi, et toute la sagesse! L'homme est «plein d'inanité et de fadaise» (III, 9, 988b et 1000 b). À quoi répond, dans le tout dernier essai, et de la toute dernière plume de Montaigne, plus que jamais fidèle à L'Ecclésiaste, où passe et repasse le vent de la vanité : 
Moi qui me vante d'embrasser si curieusement les commodités de la vie, et si particulièrement, n'y trouve, quand j’y regarde ainsi finement, à peu près que du vent. Mais quoi, nous sommes partout vent... (III, 13, 1106-1107c).

Les symboles de Montaigne sont ici le vide et le vent, comme «la bulle» de bourgeoisie romaine dont il s'amuse à se vanter aussi. Mais quoi, Aristote le disait bien, les mélancolies sont «qualités venteuses $\gg . .$.

«De la Vanité » conduit à l'image finale du «badin de la farce » qu'est l'homme qui s'ignore et qui ignore sa juste place de mortel. Mais dans ses dernières mutations, après les ultimes ajouts du texte, c'est une cruelle autodérision qui parle dans cette image, dans laquelle transparaît alors, non plus l'homme en général, mais l'écrivain luimême, berné, frustré, au seuil de la mort. C'est Montaigne qui se montre sous les traits de ce «badin », en clown risible et triste, digne d'Hamlet ou plutôt de Yorick, le clown mort de la tragédie ${ }^{336}$. La terre, la boue, le néant, le vent : tels sont les symboles de la «vanitas» dans les Essais, mais l'emblème le plus parlant, dans toute sa douloureuse mélancolie, est bien ce «badin», ce fou, qui a pour répondant, dans Hamlet, Yorick, ou plutôt mieux encore: le crâne de celui qui fut Yorick.

Le vent du néant traverse «De la Vanité» surtout dans les modulations tragiques qui l'assombrissent après 1588. Montaigne retrouve alors l'accent stoïcien qui terminait, en 1580 , «De la Solitude ». «Retirez-vous en vous-même », dit Montaigne, ici et là ; la seule voie possible est de «se contenter» de soi-même, «s'en contenter, sans désir de prolongement de vie ni de nom » (I, 39, 248a). Entendons-bien cette déclaration : sans postérité, sans gloire, et sans aucun au-delà, sans même les désirer.

C'est dans l'admirable ajout final du chapitre «Du Démentir» que Montaigne approfondit cette idée, et trouve pleinement le sens de son entreprise d'écrivain. Qu'il n'ait même aucun lecteur, qu'importe : il s'est rencontré, lui, formé, constitué :

Et quand personne ne me lira, ai-je perdu mon temps...? (II, 18, 665c)

Tout prend sens pour lui. Son livre l'a véritablement créé.

336 Voir dans Le Dernier Montaigne, le chapitre VII, « Le badin de la farce », p. 191 et 192. 
Montaigne possède une autre force. C'est dans l'austère chapitre «De la Solitude» même, qu'il reprend la devise du poète Perse: Carpamus dulcia.., "Cueillons les douceurs de la vie; nous n'avons à nous que notre vie: puis tu seras cendre et ombre, et un vain mot $[\text { fabula }]^{337}$. »

Obsessionnelle, la pensée d'Hamlet rumine son mal. Claudius l'a bien observé : et redoute

ce qu'il a d'à peu près installé dans le cœur,

Et sur quoi son cerveau bat sans cesse...

Enfermé dans le cycle de la mélancolie et du désespoir, qui se nourrissent l'un de l'autre, Hamlet souffre tant, qu'il en devient cruel : pour sa mère - «Laissez-moi vous tordre le cœur» (III.iv.34) - pour Ophélie surtout, sur l'innocence de laquelle il se venge par d'odieux sarcasmes. Hamlet se met lui-même sans cesse au bord du gouffre, et ce, non pas seulement à l'acte I sur la falaise d'Elseneur. Au bord de la folie, à force de la feindre. Au bord du mal, aussi. Il décèle en lui quelque chose de mauvais, une redoutable pulsion de destruction et de meurtre. Il en prévient Laërte, on l'a vu :

I have something in me dangerous...

(v.i.228)

S'agit-il d'une pointe de cette perversité de la «tristezza» contre laquelle Montaigne met en garde au début de «De la tristesse»?

Les Italiens ont plus sortablement baptisé de son nom la malignité (I, 2, $11 b)$

L'héritage d'Hamlet est trop lourd pour lui permettre, comme le veut Montaigne, de «savoir jouir loyalement de son être» (III, 13, 815b) : le meurtre de son père, l'infamie de sa mère, l'insolent triomphe de «ce chancre » de Claudius, usurpateur cynique et jouisseur - un doublet de Richard III - et le commandement de son père, dont il se trouve indigne. Il se ronge de culpabilité. Son monde s'est fermé, pris au piège d'un destin fatal, issu tout autant de la Nécessité antique (le rappel

337 Trad. De Pierre Villey. Texte latin : «Carpamus dulcia ; nostrum est / Quod vivis : cinis et manes et fabula fies », I, 39, B, 246 . 
d'Hécube, à l'acte II, dans la tirade du comédien, et du «pouvoir félon de la Fortune », n'est pas choisi pour rien ${ }^{338}$ ), que de la prédestination protestante. Hamlet plie devant lui : « une divinité mène à leurs fins nos plans » (v.ii.10). Le monde est irrémédiablement mauvais, Hamlet le confirme en testament à Horatio :

Et dans ce monde dur [this harsh world], prends la peine de vivre,

Pour dire mon histoire.

(v.ii.282-3)

Le mal triomphe ici sous trois espèces: le crime, le péché et la mauvaise chance. Malchance, le meurtre de Polonius - sans lequel, d'ailleurs, l'action tombait dans une impasse, peut-être. Et surtout, sens aigu du péché, qui pénètre l'univers religieux d'Hamlet, son sens du sacré : au point qu'il laisse échapper sa vengeance, en refusant de tuer Claudius en prières, ce qui conduirait ce félon tout droit en Paradis...C'est le sens du péché, complice de sa mélancolie, qui oriente l'action dramatique. Le génie de Shakespeare est d'enfermer dans un huis-clos cette problématique sans issue: les pères et les fils se répondent, de l'ouverture au dénouement, eux aussi, les Norvégiens et les Danois, les Hamlet et les Fortinbras, en chiasme ${ }^{339}$.

Montaigne, au contraire, échappe au tragique par une double « échappatoire » (c'est son mot) ${ }^{340}$. La résistance de l'œuvre, et le cours de l'écriture dirigée par le «distingo»salvateur, qui s'exerce sur la pensée, sur l'humain, sur ce monde-ci, obstinément, en refusant l'irrationnel - miracles, spectres ou sorciers, voir « Des Boiteux » ${ }^{341}$ et en laissant ostensiblement à part le surnaturel.

L'univers de la tragédie vécue par Hamlet se prolonge en deçà du monde, vers le Purgatoire et l'Enfer, et au-delà, jusqu'au ciel d'anges, dans la prière d'Horatio :

Que des essaims d'anges chantent ton requiem.

(V.ii.295)

\footnotetext{
338 II.ii.461. Cf. Montaigne, soulignant le pouvoir de l'illusion théâtrale sur le spectateur le plus cruel, cite le cas d'Alexandre, tyran de Phères, qui gémit à la représentation des malheurs d'Hécube (II, 27, 693b).

339 Sans parler des relations, elles aussi étonnantes et déterminantes, de Polonius et de Laërte.

340 «De l'Expérience », 1095 b.

${ }^{341}$ Comme Reginald Scot, Montaigne, dans l'essai «Des Boiteux», s'oppose, avec Jean Wier, au démonologue Jean Bodin. Voir G. Nakam, Les Essais, Miroir et procès de leur temps, rééd. Champion, 200 1, p. 377-392.
} 
L'univers de Montaigne se borne à l'ici-bas. Mais il est ouvert, comme son oeuvre est ouverte, libéré par l'exercice du «jugement», par la responsabilité du libre-arbitre. Il s'achève, à la dernière page, sur la lumière d'Apollon.

Hamlet est sans échappatoire, prisonnier du piège doublement tragique d'un monde coupable et d'un destin sans issue, pour rencontrer enfin la mort, à laquelle sa mélancolie aspirait.

Comme l'écrira Robert Burton, dans une vision héraclitéenne qui convient bien à son thème, « la mélancolie est une », mais :

Il y a, dans toute mélancolie, similitudo dissimilis, comme dans les visages humains, une ressemblance, et, comme dans une rivière nous nageons sur place et pourtant l'eau n'est pas identique, comme le même instrument de musique propose diverses leçons, la même maladie offre une diversité de symptômes ${ }^{342}$.

Elle est « polymorphe », dit Jackie Pigeaud (préface au Problème XXX).

Montaigne est une étape décisive dans la perception de la «mélancolie » à la Renaissance, par l'insatisfaction qui l'habite, et par le regard intérieur qui décrit ce même doute sous tant de formes : par exemple les autoportraits étonnants qui s'emboîtent l'un dans l'autre dans «De la Présomption » ${ }^{343}$. Par rapport à Shakespeare, faut-il parler d'une «évolution» historique? Peut-être, comme dans toute étude de mentalités, et de leur traduction esthétique, qu'on devrait alors, pour le premier, en raison même et d'abord de son «étrangeté », qualifier sa mélancolie de «maniériste», et pour le second, dans Hamlet, en raison des déchirements de la souffrance, et de son aura sacrée et funèbre, de «baroque»? Je ne sais. Un Jodelle (1532-1573), son humeur «noire» et souvent satanique, et son «forcènement ${ }^{344}$, sont plus proches d'un Marlowe, voire de Shakespeare, que de Ronsard ou de Montaigne ${ }^{345}$.

342 Robert Burton, Anatomie de la Mélancolie (1621), Première Partition, Section 3, Membre 1, Subdivision 2, op. cit., vol. I, p. 661.

343 Voir Le Dernier Montaigne, chap. IX, « Manières d'un autoportrait », p. 245 sq

344 «Forcènement » est le mot par lequel D’Aubigné qualifie le génie de Jodelle.

345 Évitons, pour les très grands textes, même les plus subtiles catégories. Il y a aussi un «maniérisme noir», dans lequel peut entrer Jodelle. Et je continue à tenir Montaigne pour 
La mélancolie est bien «une», il s'agit d'une même «nature ${ }^{346}$, comme le proposait Aristote, d'une «complexion», comme dit Montaigne, d'une prédisposition congénitale, révélée, comme chez Hamlet, par un choc, la mort de son père, réveillée, comme chez Montaigne, par un traumatisme comparable, la mort de La Boétie. Une «nature», donc, faite d'anxiété, d'incertitude sur son propre moi, a fortiori son moi multiple, d'in-tranquillité, dirait Pessoa, et son expression verbale et gestuelle, tendue, nerveuse, voire agitée, qui s'accompagne par intervalles d'auto-ironie, ou de la dérision trop lucide de tout et de soi, et de la vanité même. Ils sont tous deux des rejetons de «Melancholia», ou, pour reprendre le beau titre des historiens d'art Rudolf et Margot Wittkover, «enfants de Saturne ${ }^{347}$, mais dans des registres autres, avec une intensité incomparable, et surtout des trajectoires opposées, orientées qu'elles sont, l'une vers la vie, l'autre vers la mort, et par les idéologies contraires d'une liberté et d'un «fatum»: «Mon destin m'appelle», déclare Hamlet dès le début : «My fate cries out...» (I.v.58).

Devant Montaigne, Shakespeare a, me semble-t-il, une attitude ambivalente, de complicité et d'ironie, disons de complicité ironique (et d'ironie complice!). parfois il l'habille en vieux radoteur (Polonius), trop naiff (le Gonzalo du début de The Tempest). Parfois il transforme ses dires en leurs contraires, ou en sarcasmes : le cas le plus expressif est le noble «Cannibale» devenu hideux Caliban. Mais il éprouve toujours devant ce texte illustre déjà en Angleterre à la fin du siècle, une vive émotion de lecteur, et donc de créateur, il y trouve un élan de la pensée, parfois aussi du langage - idées et mots avec lesquels il joue en pirouettes et en culbutes, comme Hamlet de sa «folie».

Un Shakespeare n'a pas besoin de Montaigne pour être Shakespeare. Non pas, mais sans doute pour le devenir. Je rejoins là tout à fait l'article riche et novateur de Robert Ellrodt ${ }^{348}$. Montaigne, écrivain du «passage»- «Je ne peins pas l'être. Je peins le passage»

un grand Maniériste (et pas du tout un «baroque»). Vu les décalages dans le temps, on peut aussi parler, je le crois, d'un «maniérisme noir » pour Shakespeare.

346 Souligné par Jackie Pigeaud dans sa Postface à l'éd. de Burton citée.

347 Rudolf et Margot Wittkover, Les Enfants de Saturne, tr. Fr. Macula, Paris, 1985.

348 «Self-consciousness in Montaigne and Shakespeare», cité supra en note 6. 
- est aussi un passeur. Tous les grands livres le sont. C'est la traversée des Essais, leur médiation qui a, non seulement enrichi, mais « réfléchi » dans leur «miroir» l'univers intérieur de Shakespeare, et peut-être permis au dramaturge de reconnaître en lui et de projeter en « représentation», façonnée de bien d'autres matériaux encore, une des formes les plus nobles et les plus désespérées de sa propre «mélancolie»: Hamlet ${ }^{349}$.

Géralde NAKAM

Université de Paris III-Sorbonne Nouvelle

\footnotetext{
349 Je remercie vivement Robert Ellrodt de son observation justifiée concernant le livre qu'Hamlet tient en mains, d'autant plus que, dans le même article cité, il insistait déjà sur la qualité de l'hypothèse de Harding Craig selon laquelle ce livre serait le De Consolatione de Jérôme Cardan (Hutington Library Bulletin, 1934, p. 17-37). Je n'ai pu prendre connaissance de cet article, le vol. 1934 de H.L.B. manquant à la bibliothèque de la Sorbonne.

Mais j'ai consulté le De Consolatione, qui figure dans les Opera omnia de Cardan (éd. Lyon, 1663 en 10 tomes in fol., vol. I, fo 588-636). L'ouvrage comporte 3 livres sur la consolation des calamités, de la mort, des recours (la mort, la sagesse, la Fortune). Sur la mort, un long voyage, aussi assimilée à un rêve de voyage lointain, le rapprochement semble probant. Il est retenu dans l'éd. Norton d'Hamlet (Norton, 1963, p. 119-120). En revanche, et sauf inadvertance de ma part, on n'y trouve pas les caricatures faites par Montaigne et par Hamlet de la vieillesse. Puis-je maintenir mon hypothèse, avec réserves certes, jusqu’à plus ample informé?
} 